\title{
Comportamento de poedeiras criadas a diferentes densidades e tamanhos de grupo em ambiente enriquecido
}

\author{
Danilo Florentino Pereira( ${ }^{(1)}$, Edna dos Santos Batista(1), Filipe Teixeira Sanches ${ }^{(2)}$, \\ Luís Roberto Almeida Gabriel Filho( ${ }^{(1)}$ e Leda Gobbo de Freitas Bueno ${ }^{(2)}$
} (1)Universidade Estadual Paulista (Unesp), Campus de Tupã, Avenida Domingos da Costa Lopes, 780, CEP 17602-496 Tupã, SP,
Brasil. E-mail: danilo@tupa.unesp.br, ednasantosbatista@gmail.com, gabrielfilho@tupa.unesp.br (2)Unesp, Campus de Dracena,
Rodovia Comandante João Ribeiro de Barros, Km 651, CEP 17900-000 Dracena, SP, Brasil. E-mail: sanchesft@hotmail.com,
leda@dracena.unesp.br

Resumo - O objetivo deste trabalho foi determinar diferenças comportamentais entre poedeiras criadas sob diferentes densidades e tamanhos de grupo, em condições de ambiente enriquecido. Foram utilizadas poedeiras Isa Brown com idade entre 30 e 32 semanas alojadas em galpões de escala reduzida e distorcida. As aves foram criadas durante 28 dias, em baias com cama de maravalha, poleiro e ninho. Foram avaliados dois tamanhos de grupos (6 e 12 aves) e duas densidades de criação (774 e $1.440 \mathrm{~cm}^{2}$ por ave), em arranjo fatorial com três repetições. Em amostras de vídeo de 15 min, foram registrados as frequências e os tempos de expressão dos comportamentos: arrumar penas, banho de areia, bater asas, beber água, bicar, coçar a cabeça, ciscar, comer, empoleirar, esticar perna, perseguir, sentar e visitar o ninho. Foram observados efeitos significativos dos tratamentos e da interação entre eles. O grupo de seis aves manifestou aumento da frequência de comportamentos que indicam maior frustração das aves, independentemente da densidade. $\mathrm{O}$ tamanho de grupo é o fator mais importante para o bem-estar das aves.

Termos para indexação: bem-estar animal, etologia, sistemas de criação, zootecnia de precisão.

\section{Behavior of hens reared at different densities and group sizes in an enriched environment}

\begin{abstract}
The objective of this work was to determine the behavioral differences among laying hens reared at different densities and group sizes, in an enriched environment. Isa Brown laying hens with ages from 30 to 32-week-old, in small-sized and deformed pens, were used. Hens were raised during 28 days in bays with shavings bedding, perch and nest. Two group sizes were evaluated (6 and 12 fowls) and at two rearing densities (774 and $1,440 \mathrm{~cm}^{2}$ by fowl) in a factorial arrangement with three replicates. In fifteen-minute video footages, it was recorded the frequency and the expression period for the following behavior were recorded: feather scratching, sand bath, wing beating, drinking water, pecking, head scratching, earth scratching, eating, perching, leg stretching, pursuing, sitting, and nest visiting. The treatments and the interaction between them had significant effects. The six-hen group increased the frequency of behavior types that indicate fowl frustration, regardless of density. The group size is the most important factor for hen well-being.
\end{abstract}

Index terms: animal welfare, ethology, farming systems, precision animal production.

\section{Introdução}

A intensificação da indústria avícola durante as décadas de 1930 e 1940 fez com que a produção de ovos em gaiolas, em larga escala, se tornasse mecanizada (Singh et al., 2009). A criação em gaiolas levantou polêmicas acerca do bem-estar animal, em razão do reduzido espaço oferecido aos animais e da ausência de enriquecimento ambiental, que limitariam o repertório de atividades consideradas importantes para as aves. Além disso, algumas práticas, como a elevada densidade de criação e a debicagem, ainda são rotineiramente questionadas (Alves et al., 2007; Rios et al., 2009). A União Europeia, mercado potencial para a avicultura de postura brasileira, exige desde janeiro de 2012 (Directive 99/74/CE du Conseil du 19-07-99 concernant les volailles de l'espèce Gallus gallus élevées pour la ponte, 1999) que as aves sejam criadas com um mínimo de $550 \mathrm{~cm}^{2}$ de área por indivíduo, para gaiolas convencionais, e de $750 \mathrm{~cm}^{2}$, para gaiolas enriquecidas. Neste último tipo de gaiolas, as aves devem ter, no mínimo, $600 \mathrm{~cm}^{2}$ de área utilizável.

O mercado brasileiro lançou o protocolo de bem-estar para poedeiras em 2008 (União Brasileira 
de Avicultura, 2008). Essas ações visam atender a demanda crescente de um mercado consumidor que apoia sistemas de produção menos cruéis (Blokhuis et al., 2007; Silva et al., 2009). Aves criadas em gaiolas convencionais têm restrita capacidade de circulação e expressão de seus comportamentos naturais, o que compromete sua saúde e bem-estar (Alves et al., 2007). Neste cenário, estudos de sistemas de criação alternativos ganham importância (Bracke \& Hopster, 2006; Leone \& Estévez, 2008). Entre esses sistemas, destacam-se o de gaiolas enriquecidas, que permite às aves a expressão de comportamentos mais próximos aos naturais (Tactacan et al., 2009).

Há relatos de que a densidade de criação e o tamanho dos grupos influenciam diretamente o comportamento e desempenho de poedeiras, em gaiolas enriquecidas (Guo et al., 2012). Esses fatores têm sido bastante estudados em poedeiras, nos comportamentos de bicagem e de arranquio de penas. Zimmerman et al. (2006) e Bestman et al. (2009) verificaram que a quantidade de penas arrancadas foi maior em densidades maiores. De acordo com Keeling (1995), a disputa por recursos e a relação hierárquica são os principais fatores que influenciam o comportamento de poedeiras.

Jong et al. (2007), Pickel et al. (2010), Collins et al. (2011) e Clausen \& Riber (2012) são exemplos de autores que estudaram o comportamento das aves, em diferentes ambientes. No entanto, são poucos os trabalhos que associam densidades de criação e tamanhos de grupo na avaliação desses comportamentos. Portanto, é necessário que se estude os efeitos desses fatores de criação no comportamento das poedeiras, para que se possa propor espaços e condições de criação mais adequados.

O objetivo deste trabalho foi determinar diferenças comportamentais entre poedeiras criadas sob diferentes densidades e tamanhos de grupo, em condições de ambiente enriquecido.

\section{Material e Métodos}

A pesquisa foi realizada em dois galpões de escala reduzida e distorcida, no Campus de Tupã, da Universidade Estadual Paulista. O clima da região é do tipo Cwa, segundo classificação de Köppen, com estação seca bem definida, durante o inverno.

$\mathrm{O}$ experimento foi conduzido com três repetições, para grupos diferentes de aves, em épocas diferentes do ano. A primeira repetição foi conduzida em maio de 2010, a segunda em novembro e dezembro de 2010, e a terceira em janeiro de 2011. Cada repetição (época) durou 28 dias, e os primeiros sete foram dedicados à adaptação das aves ao novo ambiente de criação.

Em cada época, foram utilizadas 36 galinhas da linhagem Isa Brown, com idade inicial entre 30 e 32 semanas, obtidas de granjas comerciais da região. As aves foram divididas em dois tamanhos de grupos (6 e 12 aves) e criadas a duas densidades, que tiveram pequenas variações decorrentes das áreas dos boxes construídos: de 766 a $782 \mathrm{~cm}^{2}$ por ave, e de 1.426 a $1.453 \mathrm{~cm}^{2}$ por ave. Para as análises, consideraram-se os valores médios dessas densidades $\left(774 \mathrm{~cm}^{2}\right.$ ave $^{-1} \mathrm{e}$ $1.440 \mathrm{~cm}^{2} \mathrm{ave}^{-1}$ ), para caracterizar um delineamento experimental de blocos com arranjo fatorial $2 \times 2$ e três repetições.

Os galpões foram divididos em baias com ambiente enriquecido, com uso de ninho e poleiro, além da cama de maravalha em toda a área. Nas baias que receberam os grupos menores de aves (6 aves), foram dispostos dois bebedouros tipo nipple, $50 \mathrm{~cm}$ de comedouro, um ninho de $40 \times 40 \mathrm{~cm}$ e $60 \mathrm{~cm}$ de poleiro. Nas baias que receberam o grupo maior de aves (12 aves), foram dispostos quatro bebedouros tipo nipple, $100 \mathrm{~cm}$ de comedouro, um ninho de $40 \times 40 \mathrm{~cm}$ e $120 \mathrm{~cm}$ de poleiro.

Durante os ensaios, todas as aves foram submetidas ao mesmo manejo de luz, e a ração foi fornecida ad libitum. A iluminação das baias foi feita por lâmpadas incandescentes e luz natural, entre 4:00 e 21:00 h, em fotoperíodo de 17 horas de luz. No teto dos galpões, foi instalada uma minicâmera de vídeo sobre cada baia. As minicâmeras eram do modelo Mythos CCD Color (Intelbras, São José, SC), com lente de 1,5 mm para as baias menores, e de 2,5 mm, para as maiores. Essas câmeras foram conectadas à placa de captura de vídeo, instalada em computador distante cerca de $30 \mathrm{~m}$, no Laboratório de Conforto Ambiental.

Foram observados, por meio das câmeras de vídeo, os comportamentos descritos no etograma da Tabela 1 , tendo-se obtido amostras diárias de filmagem de $15 \mathrm{~min}$, durante o período da manhã (entre 9:00 e 10:00 h) e 15 min durante o período da tarde (entre 15:00 e 16:00 h). A observação dos comportamentos em 15 minutos contínuos foi proposta por Bizeray et al. (2002) e utilizada por Pereira et al. (2007) e Pereira et al. (2011). Os vídeos analisados referem-se aos três 
últimos dias de cada semana, dentro das repetições, no total de 54 vídeos por tratamento.

Os comportamentos observados tiveram suas frequências e tempos de duração registrados. Um mesmo observador, durante todo o experimento, assistiu aos vídeos, com observação simultânea de todas as aves e registro dos comportamentos individuais. Para os registros de frequências, foram calculadas as médias de ocorrências de cada comportamento nos vídeos gravados. Para os registros de tempos, a duração de cada comportamento foi estimada pela divisão do tempo de gravação (15 min) pelo número de ocorrências do respectivo comportamento.

Para o monitoramento do ambiente, foi instalado um datalogger modelo HOBO, (Onset, Bourne, MA, USA) no centro geométrico de cada galpão, com registro da temperatura de bulbo seco, da umidade relativa do ar e da temperatura de globo negro, a cada $15 \mathrm{~min}$. A partir dos dados do ambiente térmico interno dos galpões, calculou-se o índice de temperatura e umidade (ITGU), a partir da equação, ITGU $=$ Tgn $+0,36$ Tpo $-330,08$,

Tabela 1. Descrição dos comportamentos observados e analisados.

\begin{tabular}{|c|c|c|}
\hline Comportamento & Descrição & Medida \\
\hline Arrumar penas & $\begin{array}{l}\text { Atividade em que a ave limpa e arruma as } \\
\text { penas com o bico }\end{array}$ & $\begin{array}{l}\text { Frequência } \\
\text { e tempo }\end{array}$ \\
\hline Banho de areia & $\begin{array}{l}\text { Atividade em que a ave joga sobre seu corpo } \\
\text { o material da cama }\end{array}$ & $\begin{array}{l}\text { Frequência } \\
\text { e tempo }\end{array}$ \\
\hline Bater asas & Movimento em que a ave bate as duas asas & Frequência \\
\hline Beber água & $\begin{array}{l}\text { Atividade em que a ave bebe água no } \\
\text { bebedouro }\end{array}$ & $\begin{array}{l}\text { Frequência } \\
\text { e tempo }\end{array}$ \\
\hline Bicar & $\begin{array}{c}\text { Comportamento em que uma ave bica } \\
\text { qualquer parte do corpo de outra ave de } \\
\text { forma agressiva }\end{array}$ & Frequência \\
\hline Ciscar & $\begin{array}{l}\text { Atividade em que a ave procura alimento na } \\
\text { cama utilizando as patas e o bico }\end{array}$ & $\begin{array}{l}\text { Frequência } \\
\text { e tempo }\end{array}$ \\
\hline Coçar a cabeça & $\begin{array}{l}\text { Comportamento em que a ave coça a cabeça } \\
\text { com uma das patas }\end{array}$ & Frequência \\
\hline Comer & Atividade da ave comendo no comedouro & $\begin{array}{l}\text { Frequência } \\
\text { e tempo }\end{array}$ \\
\hline Empoleirar & $\begin{array}{l}\text { Comportamento em que a ave sobe e } \\
\text { permanece sobre o poleiro }\end{array}$ & $\begin{array}{l}\text { Frequência } \\
\text { e tempo }\end{array}$ \\
\hline Esticar perna & $\begin{array}{l}\text { Comportamento em que a ave estica uma asa } \\
\text { e uma perna, do mesmo hemisfério do corpo }\end{array}$ & Frequência \\
\hline $\begin{array}{l}\text { Visita ou } \\
\text { permanência no } \\
\text { ninho }\end{array}$ & $\begin{array}{l}\text { Comportamento em que a ave entra (visita) } \\
\text { e fica (permanência) dentro do ninho para } \\
\text { oviposição }\end{array}$ & $\begin{array}{l}\text { Frequência } \\
\text { e tempo }\end{array}$ \\
\hline Perseguir & $\begin{array}{l}\text { Ato em que uma ave corre atrás de outra ave } \\
\text { com intenção aparente de agressão }\end{array}$ & $\begin{array}{l}\text { Frequência } \\
\text { e tempo }\end{array}$ \\
\hline Sentar & Ato da ave de ficar sentada ou deitada & $\begin{array}{l}\text { Frequência } \\
\text { e tempo }\end{array}$ \\
\hline
\end{tabular}

em que: Tgn é a temperatura de globo negro; e Tpo é a temperatura de ponto de orvalho.

Aplicou-se o teste não paramétrico de Kruskal-Wallis para a comparação das medianas dos tratamentos. $O$ teste não paramétrico pareado de Friedman foi utilizado para comparação das variáveis bioclimáticas entre os ambientes, nas diferentes épocas. Para análise das diferenças entre os tratamentos de comportamentos de baixa ocorrência, foram elaboradas tabelas de contingência, para sistematização do número de vídeos em que apareceu determinado comportamento, de acordo com cada fator analisado. Para essas tabelas de contingência, aplicou-se o teste de qui-quadrado $\left(\chi^{2}\right)$, para verificar a dependência entre os fatores.

\section{Resultados e Discussão}

A temperatura, a umidade relativa e o índice de temperatura e umidade não diferiram entre os galpões, pelo teste de Friedman ( $p>0,05)$, durante todo o período experimental, nas três épocas de avaliação.

Os comportamentos arrumar penas, bater asas, bicar, coçar cabeça, comer, empoleirar, esticar perna e visita ao ninho apresentaram frequências diferentes de acordo com os tamanhos de grupos e com as densidades. No grupo de seis aves, esses comportamentos foram expressos de forma mais frequente (Tabela 2). Quanto às densidades de criação, os comportamentos comer e bater asas, dentro do grupo de 12 aves, foram mais frequentes na menor densidade $\left(1.440 \mathrm{~cm}^{2}\right.$ por ave).

Struelens et al. (2008) verificaram que as ocorrências de bater asas antecedem a perda de postura das aves. Lambooij et al. (1999) verificaram que frangos de corte batem mais as asas quando expostos a concentrações altas de $\mathrm{CO}_{2}$, o que indica que o aumento da ocorrência desse comportamento possivelmente está associado à diminuição do bem-estar. Esse comportamento foi mais frequente no grupo com 6 aves, o que é indicativo de menor bem-estar nesse grupo. $\mathrm{O}$ grupo com 6 aves também apresentou maior frequência no comportamento agressivo bicar, o que corrobora os relatos de D'Eath \& Kelling (2003) e Rodenburg et al. (2005), que observaram menor frequência de comportamentos agressivos em grupos maiores, pelo fato de a hierarquia nesses grupos ser baseada em uma avaliação direta de tamanho e outros sinais físicos das aves, e pelo reconhecimento individual, como ocorre em pequenos grupos. 
Para os comportamentos ciscar, visitar o ninho e empoleirar, observaram-se diferenças significativas entre as densidades apenas no grupo com 6 aves, com maior frequência de visita ao ninho na densidade de $1.440 \mathrm{~cm}^{2}$ por ave, maior frequência dos comportamentos ciscar e empoleirar na densidade de $774 \mathrm{~cm}^{2}$ por ave. Para o comportamento empoleirar, embora os valores para as duas densidades tenham sido iguais, houve diferença significativa, pelo teste não paramétrico de Kruskal-Wallis. Este teste é adequado para comparar as funções de distribuição de uma variável medida em duas amostras independentes. Dessa forma, o teste pode apontar diferenças significativas entre tratamentos com valores de medianas iguais, mas que apresentem diferentes funções de distribuição das variáveis.
Os comportamentos banho de areia e ciscar foram mais frequentes no grupo com 6 aves, mas somente na densidade $774 \mathrm{~cm}^{2}$ por ave, o que evidencia, mais uma vez, a interação entre densidade e tamanho de grupo.

Quanto à duração dos comportamentos, as aves tenderam a comer e a se sentarem por mais tempo, no grupo com 12 aves (Tabela 3). Menezes et al. (2009) afirmam que a densidades de grupos não influenciam o consumo de ração. No presente trabalho, as densidades avaliadas não interferiram no tempo de consumo, mas a frequência do comportamento comer foi maior no grupo com 12 poedeiras, independentemente da densidade. Guo et al. (2012) verificaram que poedeiras criadas à densidade menor, em ambiente enriquecido, comem menos. No presente trabalho, a duração do comportamento comer foi inversamente proporcional

Tabela 2. Medianas da frequência dos comportamentos avaliados, de acordo com o tamanho dos grupos e a densidade de criação ${ }^{(1)}$.

\begin{tabular}{|c|c|c|c|c|}
\hline \multirow[t]{2}{*}{ Comportamento } & \multicolumn{2}{|c|}{ Grupo de 6 aves } & \multicolumn{2}{|c|}{ Grupo de 12 aves } \\
\hline & $774 \mathrm{~cm}^{2}$ por ave & $1.440 \mathrm{~cm}^{2}$ por ave & $774 \mathrm{~cm}^{2}$ por ave & $1.440 \mathrm{~cm}^{2}$ por ave \\
\hline Arrumar penas & $2,17 \mathrm{~A}$ & $2,33 \mathrm{~A}$ & $1,42 \mathrm{~B}$ & $1,33 \mathrm{~B}$ \\
\hline Banho de areia & $0,25 \mathrm{~A}$ & 0,17 & $0,08 \mathrm{~B}$ & 0,08 \\
\hline Bater asas & $1,67 \mathrm{~A}$ & $1,67 \mathrm{~A}$ & $1,67 \mathrm{Bb}$ & $1,67 \mathrm{Ba}$ \\
\hline Beber água & 3,50 & 3,33 & 3,00 & 3,67 \\
\hline Bicar severamente & $0,83 \mathrm{~A}$ & $0,75 \mathrm{~A}$ & $0,25 \mathrm{~B}$ & $0,17 \mathrm{~B}$ \\
\hline Ciscar & $1,33 \mathrm{Aa}$ & $0,83 b$ & $0,50 \mathrm{~B}$ & 0,58 \\
\hline Coçar cabeça & $1,17 \mathrm{~A}$ & $1,00 \mathrm{~A}$ & $0,67 \mathrm{~B}$ & $0,42 \mathrm{~B}$ \\
\hline Comer & $3,50 \mathrm{~A}$ & $4,17 \mathrm{~A}$ & $1,83 \mathrm{Bb}$ & $2,21 \mathrm{Ba}$ \\
\hline Empoleirar & $0,17 \mathrm{Aa}$ & $0,17 \mathrm{Ab}$ & $0,08 \mathrm{~B}$ & $0,08 \mathrm{~B}$ \\
\hline Esticar perna & $0,33 \mathrm{~A}$ & $0,33 \mathrm{~A}$ & $0,08 \mathrm{~B}$ & $0,17 \mathrm{~B}$ \\
\hline Visita ao ninho & $0,17 \mathrm{Ab}$ & $0,33 \mathrm{Aa}$ & $0,17 \mathrm{~B}$ & $0,25 \mathrm{~B}$ \\
\hline Sentar & 0,17 & 0,17 & 0,33 & 0,25 \\
\hline Perseguir & 0,42 & 0,00 & 0,00 & 0,25 \\
\hline
\end{tabular}

${ }^{(1)}$ Médias seguidas de letras iguais, minúsculas entre as densidades em um mesmo grupo e maiúsculas entre os grupos a uma mesma densidade, não diferem pelo teste de Kruskal-Wallis, a 5\% de probabilidade.

Tabela 3. Medianas da duração (em segundos) dos comportamentos avaliados, de acordo com o tamanho dos grupos e a densidade de criação ${ }^{(1)}$.

\begin{tabular}{|c|c|c|c|c|}
\hline \multirow[t]{2}{*}{ Comportamento } & \multicolumn{2}{|c|}{ Grupo de 6 aves } & \multicolumn{2}{|c|}{ Grupo de 12 aves } \\
\hline & $774 \mathrm{~cm}^{2}$ por ave & $1.440 \mathrm{~cm}^{2}$ por ave & $774 \mathrm{~cm}^{2}$ por ave & $1.440 \mathrm{~cm}^{2}$ por ave \\
\hline Arrumar penas & 43,0 & 29,0 & 38,0 & 33,0 \\
\hline Banho de areia & 29,0 & 682,5 & 232,5 & 207,0 \\
\hline Beber água & 9,0 & 14,0 & 6,0 & 6,0 \\
\hline Ciscar & 53,0 & 52,0 & 56,5 & 61,0 \\
\hline Comer & $64,0 \mathrm{~B}$ & $69,0 \mathrm{~B}$ & $123,0 \mathrm{~A}$ & $95,0 \mathrm{~A}$ \\
\hline Empoleirar & 277,0 & 397,0 & $114,0 \mathrm{~b}$ & $350,5 \mathrm{a}$ \\
\hline Visita ao ninho & 96,0 & 154,0 & 162,0 & 197,0 \\
\hline Sentar & $109,5 \mathrm{~B}$ & $34,0 \mathrm{~B}$ & $329,0 \mathrm{~A}$ & $198,0 \mathrm{~A}$ \\
\hline
\end{tabular}

${ }^{(1)}$ Médias seguidas de letras iguais, minúsculas entre as densidades em um mesmo grupo e maiúsculas entre os grupos a uma mesma densidade, não diferem pelo teste de Kruskal-Wallis, a 5\% de probabilidade. 
a sua frequência de expressão. Portanto, para este comportamento, é necessário monitorar, além da frequência e do tempo de expressão, a quantidade de ração consumida pelas aves.

Struelens et al. (2008) observaram que poedeiras com acesso dificultado ao ninho expressaram de forma mais frequente os comportamentos de sentar e coçar cabeça, e gastam menos tempo em movimento. Duncan (1998) verificou que o comportamento de nidificação ocorre de uma ou duas horas antes da postura, e que as aves, quando impedidas de expressar esse comportamento, ficam frustradas e demonstram essa frustração sentando-se. Portanto, a maior frequência de visita ao ninho, no grupo com 6 aves, e o maior tempo de sentar, no grupo com 12 aves, são indícios de maior bem-estar no grupo menor. No entanto, a maior frequência de visita ao ninho, no grupo com 6 aves, pode estar associada ao fato de que, no presente trabalho, utilizou-se apenas um ninho, de dimensões iguais, em ambos os tamanhos de grupo, o que teria resultado em menor disponibilidade de ninho por ave nos grupos com 12 aves.

O comportamento empoleirar teve menor tempo de duração quando o grupo de 12 aves foi combinado com a maior densidade ( $774 \mathrm{~cm}^{2}$ por ave), e teve maior frequência no grupo de 12 aves, em ambas densidades. Guo et al. (2012) observaram que poedeiras criadas em grupos com 48 indivíduos empoleiravam mais e ficavam menos tempo no ninho do que poedeiras criadas em grupos de 21 indivíduos. No presente trabalho, os dois grupos testados podem ser considerados pequenos, quando comparados com o trabalho de Guo et al. (2012); entretanto, à densidade maior, o menor número de visitas ao ninho e o aumento das frequências de empoleirar e de ciscar indicam que, nessa densidade, há maior concorrência pelos diferentes espaços proporcionados pelo enriquecimento ambiental.

O teste de $\chi^{2}$, para os comportamentos empoleirar e perseguir, mostrou que as ocorrências desses comportamentos nos vídeos dependem da interação entre o tamanho dos grupos e as densidades de criação, e que, para a condição de 6 aves e maior densidade (774 $\mathrm{cm}^{2}$ por ave), houve mais perseguições e os animais empoleiraram-se menos (Tabela 4). É esperado que, em grupos maiores, comportamentos agressivos sejam menos frequentes (D'Eath \& Kelling, 2003; Rodenburg et al., 2005). A baixa ocorrência dos comportamentos empoleirar e perseguir, nos vídeos, está associada aos horários de preferência das aves para expressá-los. As filmagens de $15 \mathrm{~min}$ foram feitas durante os períodos da manhã (entre 9:00 e 10:00 h) e da tarde (entre 15:00 e 16:00 h). O empoleiramento é mais esperado ao entardecer e à noite (Pickel et al., 2011). Já o comportamento de perseguir está associado ao estabelecimento da hierarquia, no momento em que os indivíduos de um grupo são apresentados, o que é esperado pela manhã, quando é fornecida a primeira ração do dia.

A baixa ocorrência do comportamento sentar está associada à metodologia de coleta dos dados comportamentais. Esse comportamento foi relativamente frequente mas, na maioria das vezes, esteve

Tabela 4. Número de vídeos em que se verificaram a ocorrência dos comportamentos avaliados, e resultados do teste $\chi^{2}$ para a interação entre densidade de criação e tamanho de grupo.

\begin{tabular}{|c|c|c|c|c|c|}
\hline \multirow[t]{2}{*}{ Comportamento } & \multicolumn{2}{|c|}{ Grupo de 6 aves } & \multicolumn{2}{|c|}{ Grupo de 12 aves } & \multirow{2}{*}{$\begin{array}{c}\mathrm{p} \text { do teste } \\
\chi^{2}\end{array}$} \\
\hline & $774 \mathrm{~cm}^{2}$ por ave & $1.440 \mathrm{~cm}^{2}$ por ave & $774 \mathrm{~cm}^{2}$ por ave & $1.440 \mathrm{~cm}^{2}$ por ave & \\
\hline Arrumar penas & 53 & 54 & 53 & 54 & 0,946 \\
\hline Banho de areia & 8 & 4 & 15 & 15 & 0,327 \\
\hline Bater asas & 36 & 32 & 35 & 33 & 0,994 \\
\hline Beber água & 53 & 54 & 53 & 54 & 1,000 \\
\hline Bicar severamente & 28 & 20 & 15 & 21 & 0,130 \\
\hline Ciscar & 47 & 47 & 46 & 49 & 0,828 \\
\hline Coçar cabeça & 17 & 17 & 17 & 18 & 0,906 \\
\hline Comer & 53 & 54 & 53 & 54 & 1,000 \\
\hline Empoleirar & 2 & 21 & 14 & 21 & $0,009^{*}$ \\
\hline Esticar perna & 30 & 17 & 14 & 16 & 0,138 \\
\hline Visita ao ninho & 32 & 49 & 34 & 41 & 0,462 \\
\hline Sentar & 12 & 13 & 13 & 27 & 0,211 \\
\hline Perseguir & 8 & 3 & 0 & 3 & $0,024 *$ \\
\hline
\end{tabular}

*Interação significativa entre os tratamentos, pelo teste de $\chi^{2}$, a $5 \%$ de probabilidade. 
associado a outros comportamentos. De acordo com a metodologia utilizada, apenas o comportamento ativo (arrumar as penas, por exemplo) era registrado. Os comportamentos esticar perna e coçar a cabeça são comportamentos relatados como menos frequentes, $\mathrm{o}$ que esteve de acordo com os resultados coletados no presente trabalho. O comportamento de banho de areia foi pouco observado nos vídeos. Jong et al. (2007) verificaram que poedeiras, quando há livre escolha entre diferentes substratos de cama, não expressam o banho de areia com regularidade nos substratos areia e maravalha, e verificaram a preferência dessas aves pela turfa. Olsson et al. (2002) relatam que, na natureza, as aves preferem expressar banho de areia na terra. A baixa ocorrência observada nos vídeos do banho de areia deve estar relacionada ao substrato utilizado. Constatada a importância desse comportamento para o bem-estar das aves, o resultado observado remete à necessidade de outros estudos sobre substratos de cama que favoreçam esse tipo de comportamento.

\section{Conclusões}

1. A densidade e o tamanho do grupo afetam as expressões dos comportamentos das aves em ambiente enriquecido.

2. Comportamentos que indicam maior frustração das aves, como bicar severo e bater asas, são expressados mais frequentemente em grupos menores de aves, independentemente da densidade de criação.

3. O tamanho de grupo é o fator mais importante para o bem-estar das aves, mas a densidade pode afetar essa condição.

\section{Agradecimentos}

Ao Conselho Nacional de Desenvolvimento Científico e Tecnológico (CNPq) e à Fundação de Amparo à Pesquisa do Estado de São Paulo (Fapesp), por auxílio financeiro; e à Granja Kakimoto, pela aves cedidas para o experimento.

\section{Referências}

ALVES, S.P.; SILVA, I.J.O. da; PIEDADE, S.M. de S. Avaliação do bem-estar de aves poedeiras comerciais: efeitos do sistema de criação e do ambiente bioclimático sobre o desempenho das aves e a qualidade dos ovos. Revista Brasileira de Zootecnia, v.36, p.1388-1394, 2007. DOI: 10.1590/S1516-35982007000600023.
BESTMAN, M.; KOENE, P.; WAGENAAR, J.-P. Influence of farm factors on the occurrence of feather pecking in organic reared hens and their predictability for feather pecking in the laying period. Applied Animal Behaviour Science, v.121, p.120-125, 2009. DOI: 10.1016/j.applanim.2009.09.007.

BIZERAY, D.; ESTEVEZ, I.; LETERRIER, C.; FAURE, F.M. Effects of increasing environmental complexity on the physical activity of broiler chickens. Applied Animal Behaviour Science, v.79, p.27-41, 2002. DOI: 10.1016/S0168-1591(02)00083-7.

BLOKHUIS, H.J.; NIEKERK, T.F. van; BESSEI, W.; ELSON, A.; GUÈMENÈ, D.; KJAER, J.B.; LEVRINO, M.G.A.; NICOL, C.J.; TAUSON, R.; WEEKS, C.A.; WEERD, H.A. van der. The LayWel project: welfare implications of changes in production systems for laying hens. World's Poultry Science Journal, v.63, p.101-114, 2007. DOI: 10.1079/WPS2006132.

BRACKE, M.B.M.; HOPSTER, H. Assessing the importance of natural behavior for animal welfare. Journal of Agricultural and Environmental Ethics, v.19, p.77-89, 2006. DOI: 10.1007/ s10806-005-4493-7.

CLAUSEN, T.; RIBER, A.B. Effect of heterogeneity of nest boxes on occurrence of gregarious nesting in laying hens. Applied Animal Behaviour Science, v.142, p.168-175, 2012. DOI: 10.1016/j.applanim.2012.10.005.

COLLINS, L.M.; ASHER, L.; PFEIFFER, D.U.; BROWNE, W.J.; NICOL, C.J. Clustering and synchrony in laying hens: the effect of environmental resources on social dynamics. Applied Animal Behaviour Science, v.129, p.43-53, 2011. DOI: 10.1016/j. applanim.2010.10.007.

D'EATH, R.B.; KEELING, L.J. Social discrimination and aggression by laying hens in large groups: from peck orders to social tolerance. Applied Animal Behaviour Science, v.84, p.197-212, 2003. DOI: 10.1016/j.applanim.2003.08.010.

DIRECTIVE 99/74/CE du Conseil du 19-07-99 concernant les volailles de l'espèce Gallus gallus élevées pour la ponte. Journal Officiel des Communautés Européennes, JO L:203/53, p.53-57, 1999.

DUNCAN, I.J. Behavior and behavioral needs. Poultry Science, v.77, p.1766-1772, 1998.

GUO, Y.Y.; SONG, Z.G.; JIAO, H.C.; SONG, Q.Q.; LIN, H. The effect of group size and stocking density on the welfare and performance of hens housed in furnished cages during summer. Animal Welfare, v.21, p.41-49, 2012.

JONG, I.C. de; WOLTHUIS-FILLERUP, M.; REENEN, C.G. van. Strength of preference for dustbathing and foraging substrates in laying hens. Applied Animal Behaviour Science, v.104, p.24-36, 2007. DOI: 10.1016/j.applanim.2006.04.027.

KEELING, L. Spacing behaviour and an ethological approach to assessing optimum space allocations for groups of laying hens. Applied Animal Behaviour Science, v.44, p.171-186, 1995. DOI: 10.1016/0168-1591(95)00612-V.

LAMBOOIJ,E.; GERRITZEN, M.A.; ENGEL, B.; HILLEBRAND, S.J.W.; LANKHAAR, J.; PIETERSE, C. Behavioural responses during exposure of broiler chickens to different gas mixtures. Applied Animal Behaviour Science, v.62, p.255-265, 1999. DOI: 10.1016/S0168-1591(98)00214-7. 
LEONE, E.H.; ESTÉVEZ, I. Economic and welfare benefits of environmental enrichment for broiler breeders. Poultry Science, v.87, p.14-21, 2008. DOI: 10.3382/ps.2007-00154.

MENEZES, P.C. de; CAVALCANTI, V.F.T.; LIMA, E.R. de; EVÊNCIO NETO, J. Aspectos produtivos e econômicos de poedeiras comerciais submetidas a diferentes densidades de alojamento. Revista Brasileira de Zootecnia, v.38, p.2224-2229, 2009. DOI: 10.1590/S1516-35982009001100023.

OLSSON, I.A.S.; DUNCAN, I.J.H.; KEELING, L.; WIDOWSKI, T.M. How important is social facilitation for dustbathing in laying hens. Applied Animal Behaviour Science, v.79, p.285-297, 2002. DOI: 10.1016/S0168-1591(02)00117-X.

PEREIRA, D.F.; NÄÄS, I.A.; SALGADO, D.D.; GASPAR, C.R.; BIGHI, C.A.; PENHA, N.L.J. Correlations among behavior, performance and environment in broiler breeders using multivariate analysis. Brazilian Journal of Poultry Science, v.9, p.207-213, 2007.

PEREIRA, D.F.; OLIVEIRA, S.C. de; PENHA, N.L.J. Logistic regression to estimate the welfare of broiler breeders in relation to environmental and behavioral variables. Engenharia Agrícola, v.31, p.33-40, 2011. DOI: 10.1590/S0100-69162011000100004.

PICKEL, T.; SCHOLZ, B.; SCHRADER, L. Perch material and diameter affects particular perching behaviours in laying hens. Applied Animal Behaviour Science, v.127, p.37-42, 2010. DOI: 10.1016/j.applanim.2010.08.005.

PICKEL, T.; SCHOLZ, B.; SCHRADER, L. Roosting behaviour in laying hens on perches of different temperatures: trade-offs between thermoregulation, energy budget, vigilance and resting. Applied Animal Behaviour Science, v.134, p.164-169, 2011. DOI: 10.1016/j.applanim.2011.07.003.

RIOS, R.L.; BERTECHINI, A.G.; CARVALHO, J.C.C.; CASTRO, S.F.; COSTA, V.A. Effect of cage density on the performance of 25to 84-week-old laying hens. Revista Brasileira de Ciência Avícola, v.11, p.257-262, 2009. DOI: 10.1590/S1516-635X2009000400007.
RODENBURG, T.B.; KOENE, P.; BOKKERS, E.A.M.; BOS, M.E.H.; UITDEHAAG, K.A.; SPRUIJT, B.M. Can short-term frustration facilitate feather pecking in laying hens? Applied Animal Behaviour Science, v.91, p.85-101, 2005. DOI: 10.1016/j. applanim.2004.08.023.

SILVA, R.B.T.R. da; NÄÄS, I. de A.; MOURA, D.J. de. Broiler and swine production: animal welfare legislation scenario. Scientia Agricola, v.66, p.713-720, 2009. DOI: 10.1590/ S0103-90162009000600001.

SINGH, R.; CHENG, K.M.; SILVERSIDES, F.G. Production performance and egg quality of four strains of laying hens kept in conventional cages and floor pens. Poultry Science, v.88, p.256-264, 2009. DOI: 10.3382/ps.2008-00237.

STRUELENS, E.; VAN NUFFEL, A.; TUYTTENS, F.A.M.; AUDOORN, L.; VRANKEN, E.; ZOONS, J.; BERCKMANS, D.; ÖDBERG, F.; DONGEN, S. van; SONCK, B. Influence of nest seclusion and nesting material on pre-laying behaviour of laying hens. Applied Animal Behaviour Science, v.112, p.106-119, 2008. DOI: 10.1016/j.applanim.2007.07.010.

TACTACAN, G.B.; GUENTER, W.; LEWIS, N.J.; RODRIGUEZ-LECOMPTE, J.C.; HOUSE, J.D. Performance and welfare of laying hens in conventional and enriched cages. Poultry Science, v.88, p.698-707, 2009. DOI: 10.3382/ps.2008-00369.

UNIÃO BRASILEIRA DE AVICULTURA. Protocolo de bem-estar para aves poedeiras. São Paulo: União Brasileira de Avicultura, 2008. 23p. Disponível em <http://www.abef.com.br/ uba/arquivos/protocolo_de_bem_estar_para_aves_poedeiras final_11_07_08.pdf $>$. Acesso em: 20 fev. 2013.

ZIMMERMAN, P.H.; LINDBERG, A.C.; POPE, S.J.; GLENA, E.; BOLHUIS, E; NICOL, C.J. The effect of stocking density, flock size and modified management on laying hen behaviour and welfare in a non-cage system. Applied Animal Behaviour Science, v.101, p.111-124, 2006. DOI: 10.1016/j.applanim.2006.01.005.

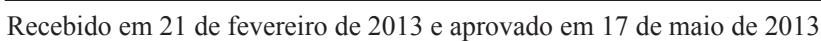

